\title{
Evaluación del impacto de los pilares de la seguridad y soberanía alimentaria y nutricional SSAN en las familias de la comunidad la pavana, municipio de Somotillo-2014
}

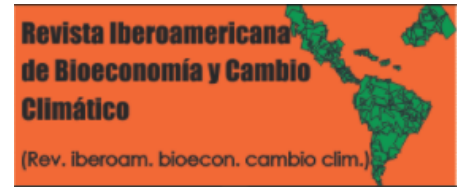

\author{
Evaluation of the impact of the pillars of SSAN food and \\ nutrition security and sovereignty in the families of the la \\ pavana community, Somotillo municipality-2014
}

\author{
Sáenz Rojas, Silvio Javier; Rivas García, Julio Antonio; Paredes \\ Castillo, Lannier Alberto; Catín Chiong, Adrián Humberto; Trouño, \\ Editor Academico Prof. Dr. Pedro Jose
}

\author{
Silvio Javier Sáenz Rojas \\ silviosaenz@yahoo.com \\ UNAN- León, CUR-Somotillo., Nicaragua \\ Julio Antonio Rivas García \\ UNAN- León, CUR-Somotillo., Nicaragua \\ Lannier Alberto Paredes Castillo \\ UNAN- León, CUR-Somotillo., Nicaragua \\ Adrián Humberto Catín Chiong \\ UNAN- León, CUR-Somotillo., Nicaragua \\ Editor Academico Prof. Dr. Pedro Jose Trouño \\ Universidad Nacional Autónoma de Nicaragua, \\ León. Escuela de Ciencias Agrarias y Veterinarias. \\ Departamento de Agroecología, Nicaragua
}

\section{Revista Iberoamericana de Bioeconomía y Cambio Climático \\ Universidad Nacional Autónoma de Nicaragua, León, Nicaragua ISSN-e: 2410-7980 \\ Periodicidad: Semestral \\ vol. 4, núm. 8, 2018 \\ czuniga@ev.unanleon.edu.ni}

Recepción: 15 Febrero 2018

Aprobación: 24 Octubre 2018

URL: http://portal.amelica.org/ameli

jatsRepo/394/3941755009/index.html

DOI: https://doi.org/10.5377/ribcc.v4i8.6647

Autor de correspondencia: silviosaenz@yahoo.com
Resumen: El estudio permite identificar la situación de la Seguridad Alimentaria (Bioeconomía Biológica); disponibilidad, acceso, consumo y aprovechamiento biológico de los alimentos de las familias rurales de la comunidad La Pavana del municipio de Somotillo. La Investigación es descriptiva, se realizaron la aplicación de entrevistas y 90 encuestas semiestructuradas. Los resultados evidencian que el $21 \%$ de las familias cuentan con terreno propio para la producción de cultivos las familias no disponen de variedad de alimentos todo el año, no hay una diversidad productiva. El patrón alimentario está conformado por 21 alimentos para el departamento de Chinandega, solo 9 alimentos son consumidos en la dieta de las familias: Café, azúcar, sal, aceite, pollo, tortilla de maíz, frijol, arroz y huevos. El tiempo de comida de acuerdo a sus ingresos económicos y debido a que estos son bajos el $13 \%$ realizan un tiempo de comida, el 28\%, dos tiempos y un $59 \%$ consume sus alimentos tres veces al día. El $41 \%$ de las familias se encuentra en insuficiencia critica del consumo de alimentos, el $51 \%$ es insuficiente debido al bajo consumo de frutas y verduras demostrando un deterioro en el estado de salud y nutricional incidiendo en la utilización biológica de los alimentos, evidenciando un 3\% de desnutrición de niños en la comunidad, influye en la presencia de enfermedades respiratorias y diarreicas con un $26 \%$ y $32 \%$. Los ingresos familiares el promedio fue de 2.60 dólares por día, logrando cubrir un $17.27 \%$ del costo de la canasta básica urbana y un $21.59 \%$ de la canasta rural.

Palabras clave: Disponibilidad, Acceso, Consumo, Aprovechamiento biológico de los alimentos.

Abstract: The study allows to identify the situation of Food Security (Biological Bioeconomy) ; availability, access, consumption and biological use of food from rural families in the La Pavana community in the municipality of Somotillo. The research is descriptive, interviews were conducted and 90 semi-structured surveys were conducted. The results show that $21 \%$ of the families have their own land for the production of crops, families do not have a variety of food all year round, 
there is no productive diversity. The food pattern is made up of 21 foods for the department of Chinandega, only 9 foods are consumed in the families' diet: coffee, sugar, salt, oil, chicken, corn tortilla, beans, rice and eggs. The meal time according to their income and because these are low $13 \%$ make a meal time, $28 \%$, two times and $59 \%$ consume their food three times a day. $41 \%$ of families are in critical failure of food consumption, $51 \%$ is insufficient due to low consumption of fruits and vegetables demonstrating a deterioration in health and nutritional status affecting the biological use of food, evidencing a $3 \%$ of malnutrition of children in the community, influences the presence of respiratory and diarrheal diseases with $26 \%$ and $32 \%$. The average family income is 2.60 dollars per day, managing to cover $17.27 \%$ of the cost of the urban basic basket and $21.59 \%$ of the rural basket.

Keywords: Availability, Access, Consumption, Biological use of food.

\section{INTRODUCCIÓN}

La seguridad alimentaria es un tema de gran relevancia (Paola, 2008), principalmente en países con gran inequidad económica en donde es muy importante prestar atención no sólo a la seguridad alimentaria a nivel nacional sino también a niveles más desagregados (Amuninic, 2012).

Dada la complejidad del problema de seguridad alimentaria, en esta investigación se abordaron los cuatros pilares de la seguridad alimentaria: la disponibilidad agregada de los alimentos, el acceso de los hogares a los alimentos, el consumo de los alimentos y el aprovechamiento biológico de los alimentos (FAO, 2009).

El plan de Desarrollo Humano 2008-2012, Bonilla, (2001) considera la seguridad y soberanía alimentaria donde plantea que en Nicaragua el hambre y la desnutrición están asociadas a la pobreza extrema , cita que “ La seguridad alimentaria garantizará el derecho a la tierra y productos nacionales, especialmente los MIPYMES rurales, uso de las aguas superficiales para riego, reducir la vulnerabilidad, el fortalecimiento de la equidad en la distribución a precios justos y garantizar la equidad de género, la sostenibilidad de la producción agropecuaria y la eficiencia de la industria alimentaria nacional (PMA, 2009). (Error 1: La referencia PMA, 2009 debe estar ligada) (Error 2: El tipo de referencia PMA, 2009 es un elemento obligatorio) (Error 3: No existe una URL relacionada)

El presente estudio tuvo como propósito, analizar la situación de seguridad alimentaria de las familias en la comunidad de La Pavana y sus sectores vecinos (Los Balcones, Santa Ana, Los Arados) del municipio de Somotillo, departamento de Chinandega durante el período de Abril - diciembre 2014, la cual tiene una población de 116 familias.

América Latina y el Caribe es la única región que previo a la crisis alimentaria había logrado reducir el hambre (Brunetti, 2009). Sin embargo, tras el aumento en el nivel de los precios de alimentos y la subsiguiente crisis económica, esta tendencia se revierte, implicando que la seguridad alimentaria tomara relevancia en la agenda política y social de los países de la región, a nivel subregional y regional (Ramos, 2008). Para una región que presenta un superávit en la disponibilidad de alimentos, las últimas estimaciones de FAO (2006)

\section{NotAS DE AUTOR}


indican que la población subnutrida en América Latina y el Caribe alcanza los 52,5 millones de personas, equivalentes a un $9 \%$ de su población y a 600 mil personas menos que en el 2009.

\section{Diseño Metodológico}

\section{Enfoque metodológico:}

El enfoque metodológico que se utilizó en el estudio de esta investigación es cuali-cuantitativo, dado que primeramente se realizó una indagación a través de la aplicación de entrevistas a las personas que dirigen las instituciones que están relacionado con éste, las cuales son: MAGFOR, Alcaldía municipal y MINSA. Posteriormente se aplicó una encuesta a las familias que habitan en la Comunidad La Pavana y los sectores aledaños a ella (Delgadillo, 2004).

\section{Población y muestra}

El universo de estudio para esta investigación, estuvo constituido por tres autoridades que integran la Comisión Municipal de soberanía y Seguridad Alimentaria Nutricional (COMUSSAN), integrado por el Ministerio Agropecuario y Forestal (MAGFOR), alcaldía municipal de Somotillo y el Ministerio de Salud (MINSA) quienes son los responsables de los programas de seguridad alimentaria y nutricional del municipio y además 116 familias que son habitantes de la comunidad la pavana y sus sectores aledaños : Los Balcones, Santa Ana Y Los Arados.

Para la selección de la muestra en el caso de las autoridades responsables de los programas de seguridad alimentaria y nutricional del municipio, se tomó en cuenta a los responsables de las instituciones antes mencionadas por ser considerados informantes claves.

En lo referido a las familias, se tomó como población 116 familias que habitan en la Comarca: La Pavana, Los Balcones, Santa Ana Y Los Arados. Para el cálculo del tamaño de la muestra se utilizó la formula estadística, a continuación, se describe el significado de cada una de las variables que le integran (Hernández, 2006).

$$
n=\frac{N^{*} Z_{\alpha}^{2 *} p^{*} q}{E^{2 *}(N-1)+Z_{\alpha}^{2 *} p^{*} q}
$$

[tamaño de la población]

$\mathrm{N}=$ tamaño de la población

$\mathrm{Z}=$ nivel de confianza

$\mathrm{P}=$ probabilidad de éxito, o proporción esperada

$\mathrm{q}=$ probabilidad de fracaso

$\mathrm{E}=$ precisión (error máximo admisible en termino de proporción).

Para el cálculo del tamaño $\mathrm{n}$ de la muestra en este estudio, los valores que se asignaron a cada una de las variables son los siguientes:

$\mathrm{N}=116$

$\mathrm{Z}=1.96$

$\mathrm{P}=0.5$

$\mathrm{Q}=0.5$

$\mathrm{E}=0.05$

Dando como resultado una muestra de tamaño $\mathrm{n}=89$ familias, a las que se les aplicó la encuesta. 
Para la aplicación de la encuesta se utilizó el muestreo aleatorio estratificado, dado que la población entre los sectores no es homogénea con relación a la característica que se desea estudiar: disponibilidad, acceso, consumo y aprovechamiento biológico de los alimentos.

Para determinar el tamaño de la muestra en cada estrato se utilizó el método cálculo proporcional al tamaño del estrato, el cual establece que debe de existir una relación proporcional entre el tamaño del estrato y el número de elementos que aporta a la muestra. Cuanto mayor sea el estrato, mayor será el tamaño de la muestra seleccionada. La tabla No 2. Muestra la cantidad de encuesta que se aplicó en cada sector.

TABLA NO 2

Muestra la cantidad de encuesta que se aplicó en cada sector.

\begin{tabular}{|c|c|c|c|}
\hline Sectores & Cantidad de familia & $\mathbf{\%}$ & No. Encuesta \\
\hline La Pavana & 58 & $50 \%$ & 45 \\
\hline Santa Ana & 20 & $17 \%$ & 15 \\
\hline Los Balcones & 32 & $28 \%$ & 25 \\
\hline Los Arados & 6 & $5 \%$ & 5 \\
\hline Total & $\mathbf{1 1 6}$ & $\mathbf{1 0 0 \%}$ & $\mathbf{9 0}$ \\
\hline
\end{tabular}

\section{Métodos y técnicas a utilizar para la recolección y análisis de datos.}

Para la recolección de la información en esta investigación se utilizó como técnicas la entrevista y la encuesta.

Con la aplicación de la entrevista se obtuvo información relevante sobre la situación de seguridad alimentaria en la comunidad (La Pavana), tales como la existencia de programas alimenticios, nivel de aceptación que estos tienen en la comunidad, resultados obtenido con la aplicación de programas alimentarios, si se ha logrado reducir la pobreza, si existe coordinación entre las instituciones vinculadas a la situación de seguridad alimentaria en la comunidad.

En el caso de la encuesta se tomó en cuenta los cuatro pilares de la seguridad alimentaria; disponibilidad, acceso, consumo y aprovechamiento biológico de los alimentos.

\section{Procesamiento y análisis de la información}

Para el procesamiento y análisis de la información en el caso de las entrevistas, primeramente, se agrupó en una tabla de doble entrada las respuestas de los entrevistados de acuerdo a cada pregunta. Luego se identificaron por pregunta las afirmaciones en que coincidieron o no los entrevistados, representándolo en porcentaje para su posterior análisis.

En el caso de la encuesta, el primer paso que se utilizó en el procesamiento y análisis de los datos, es la codificación, una vez codificados los datos, se procedió a elaborar una base de datos usando el programa Statiscal Packageforthe Social Sciences 21 (SPSS).

\section{Fuentes de la investigación}

Usualmente se habla de dos tipos de fuente de recolección de información: las primarias y las secundarias. 
En el caso de esta investigación, las fuentes primarias la constituyeron las autoridades miembros de la COMUSSAN y las familias que habitan en la comunidad La Pavana del municipio de Somotillo. Y como fuente secundaria se utilizó la ley No. 693. Ley de Soberanía y Seguridad Alimentaria y Nutricional, revistas sobre seguridad alimentaria, documentos impresos y digitales que se consultaron sobre aspectos relevantes relacionados con esta investigación.

\section{Variables}

Tabla 4 Ver anexos

\section{Resultados}

ENCUESTA a familias habitantes de la comunidad La Pavana Municipio de Somotillo.

\section{Disponibilidad}

Tipo de Actividad Económica que realiza en el ciclo anual los habitantes de la comunidad La Pavana. En cuanto a la actividad económica que realizan en el ciclo anual, el gráfico $\mathrm{N}^{\circ} 1$ muestra que el $56 \%$ de los encuestados se dedican a la agricultura, $21 \%$ se dedican tanto a la agricultura como a la ganadería, el $1 \%$ a la actividad agrícola, pecuaria y forestal, y un $22 \%$ se dedican a otras actividades tales como; Triciclero, albañil, carpintero, alfarero, lavado y planchado. La mayor parte de la población se dedica a la agricultura y ganadería en pequeña escala ya que no cuentan con áreas disponibles para el establecimiento de cultivos y la producción es destinada para autoconsumo y esta no cubre la demanda de la población, por lo tanto buscan otras fuentes de ingresos en la cabecera municipal.

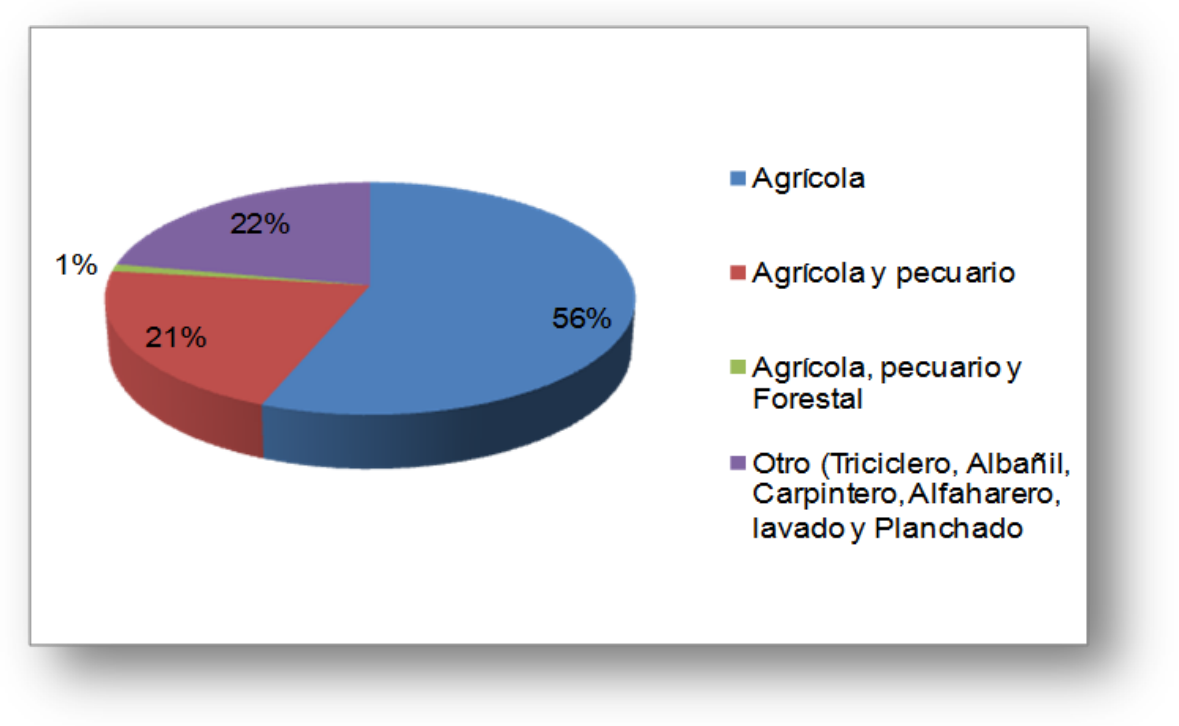

GRÁFICO No 1

Actividad económica que realizan.

Según el gráfico No 2. de los rubros que se establecen en la época de primera se encontró que el 33\% de los encuestados siembran maíz, el $4 \%$ maíz y sorgo, el $2 \%$ maíz y frijol alacín, el $1 \%$ maíz, frijol alacín y sorgo, un $60 \%$ expresó que no siembran, de estos un $22 \%$ no se dedican a la agricultura ya que realizan otras actividades 
tales como (albañil, carpintero, jornalero, triciclero, pulperos, negociante ambulante, alfarero) durante el año. Es importante resaltar que el rubro principal que más siembran es el maíz en asocio con el cultivo del frijol o el sorgo los cuales son considerados como alimentos principales en su dieta alimenticia esto a raíz de costumbre y cultura de los que accesan a cultivar para su auto consumo a demás no cuentan con los recursos necesarios para poder aplicar a extensión de tierras para poder producir.

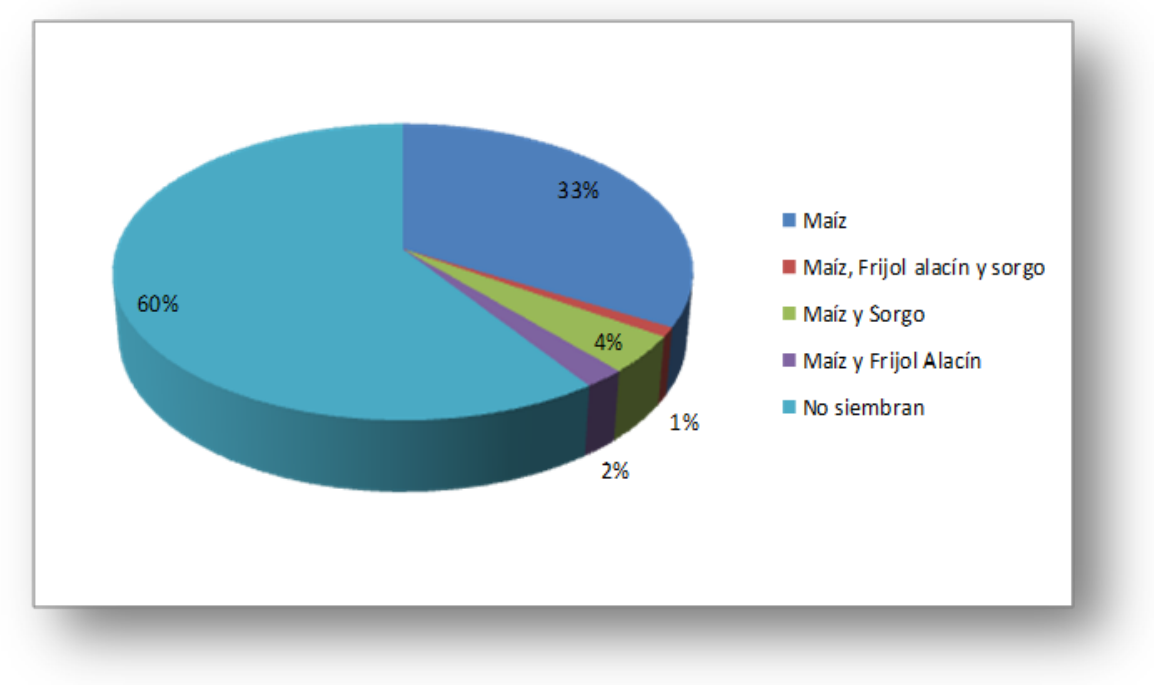

GRÁFICO NO 2.

Rubros que se establecen en la época de primera.

Tipos de rubros que se establecen en la comunidad LA PAVANA

TABLA NO 1

Tipos de rubros.

\begin{tabular}{cccc}
\hline La Pavana & Los Balcones & Santa Ana & Los Arados \\
\hline MAIZ & MAIZ & MAIZ & MAIZ \\
SORGO & SORGO & AJONJOLI & AJONJOLI \\
FRIJOL ALACIN & AJONJOLI & SORGO & SORGO \\
PIPIAN/ PIPIAN & FRIJOL ALAZIN & FRIJOL ALAZIN & \\
AJONJOLI & & PIPIAN/ AYOTE & \\
TOMATE & & & \\
\hline
\end{tabular}

Encuesta comunidad La Pavana

Los rubros que mayormente producen ESTE SECTOR son: el maíz, ajonjolí y sorgo, ya que estos tienen una buena producción que se adaptan a las condiciones edafo-climáticas de la zona, son los que generan mayor ingreso y tienen más área de siembra. Los otros rubros los productores los siembran para complementar el plato alimenticio por ser productos a los que no tienen alcance de adquirir en los mercados (Tabla No. 1). 


\section{Producción Interna de alimentos.}

Rendimiento en quintales de granos básicos por manzanas en las familias encuestadas de la comunidad los Limones. Ver Gráfico No. 3

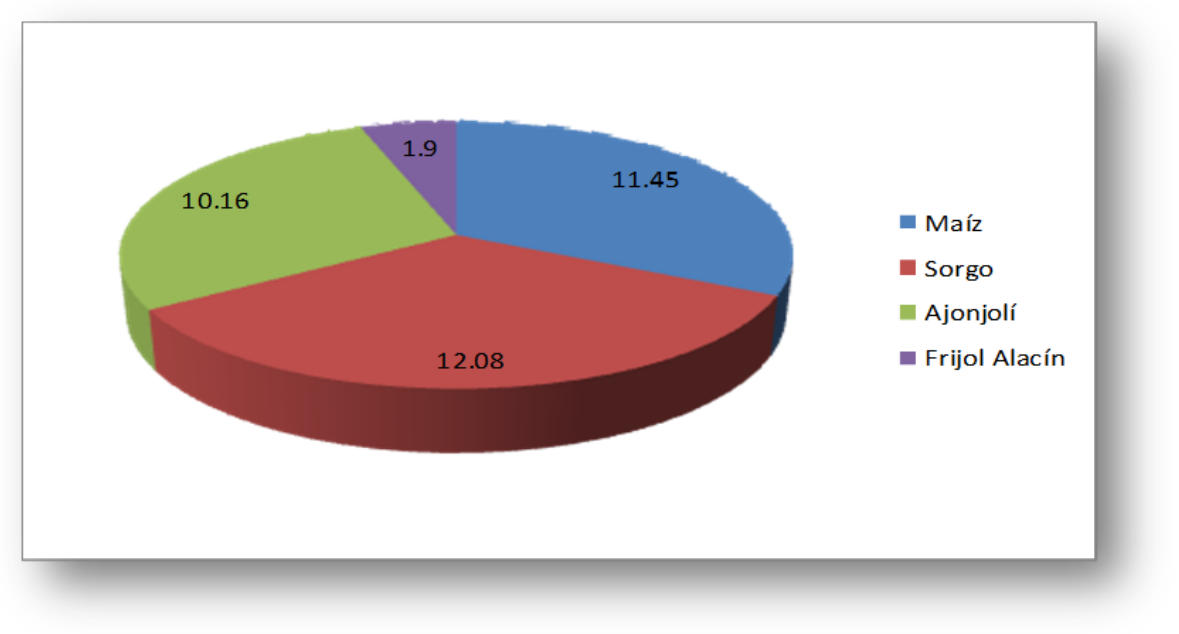

\section{GRÁFICO NO. 3 \\ Rendimiento en qq/Mz de granos básicos. MAGFOR}

Según datos del MAGFOR (2009) del municipio, el promedio de producción de maíz es de 10 a 12 quintales por manzana por debajo del promedio nacional en la zona seca que es de $20 \mathrm{qq} / \mathrm{mz}$, en sorgo de 13 a 14 quintales por manzana, en ajonjolí de 8 quintales por manzana, y en el cultivo de frijol alacín no hay datos específicos ya que este cultivo se consume en legumbre.

De acuerdo a los encuestados se encontró que el promedio de producción en maíz es de 11.45 quintales por manzana, en sorgo es de 12.08 quintales, 10.16 quintales de ajonjolí y frijol alacín es de 1.9 quintales por manzana el cual es cosechado para semilla, dado que este rubro lo consumen como legumbre, para mejorar el plato alimentario las familias consumen 1.22 docenas de ayote y pipián en promedio, el bajo rendimiento de estos cultivos se debe al manejo tradicional y muchas veces por la baja fertilidad del suelo ya que los productores no tienen la capacidad para obtener el fertilizante y realizar las aplicaciones de fertilizantes en tiempos adecuados. 
Atención a la seguridad alimentaria.

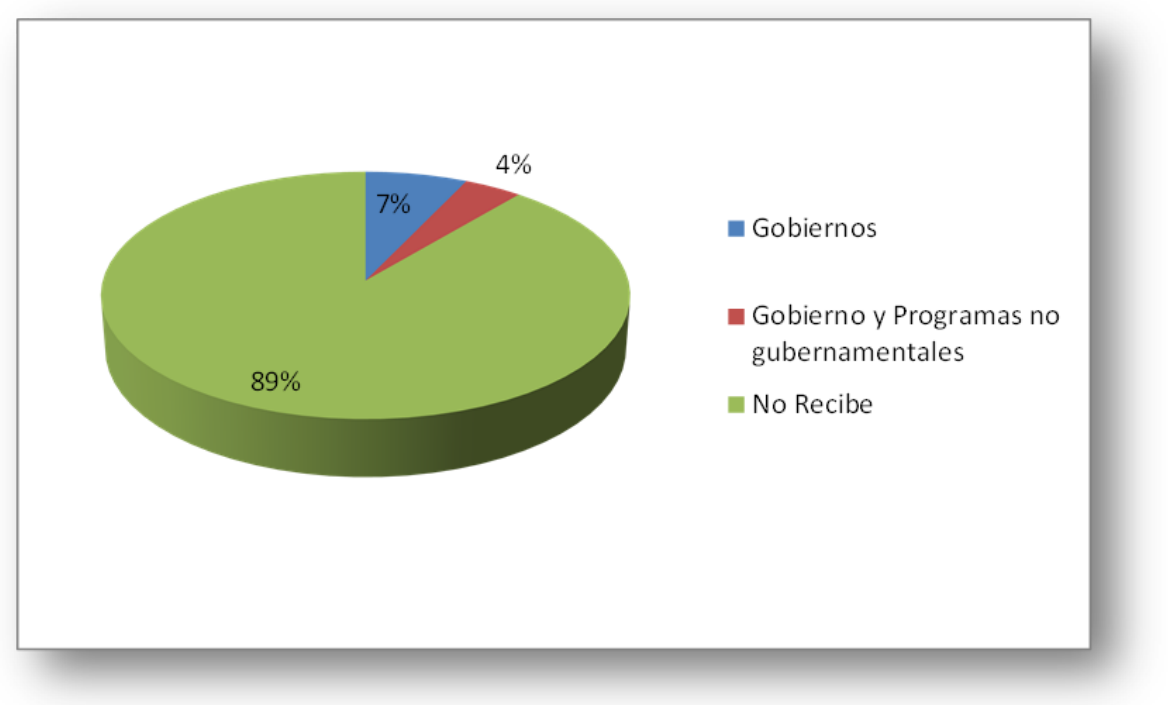

GRÁFICO NO. 4

Cantidad de familia que reciben ayuda alimentaria. INTA

El Gráfico No 4. muestra el resultado de la ayuda alimentaria en el cual se encontró que el 7\% recibe ayuda alimenticia por parte del gobierno, $4 \%$ recibe la ayuda por parte del Gobiernos y Programas no gubernamentales y el $89 \%$ no recibe, según INTA esto es debido a las dificultades de organización de los habitantes de la comunidad y a preferencias de los lideres ya que está regido por políticas de gobierno.

\section{Acceso}

Técnicas de obtención de los alimentos

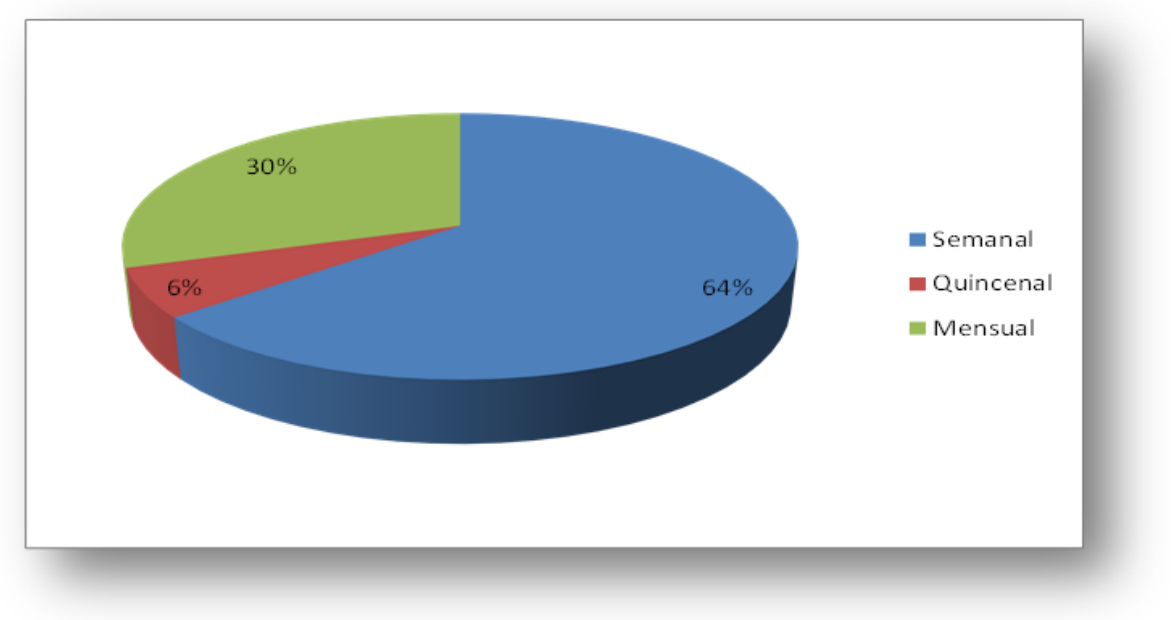

GRÁFICO NO. 5

Frecuencia de realización de compra de alimentos.

INTA 
En las familias encuestadas se encontró que el 64\% compra los alimentos semanales, el $30 \%$ mensual, el $6 \%$ quincenal. Esto demuestra que las compras de los alimentos la realizan con mayor frecuencia semanal ya que no hay un ingreso fijo y lo que ganan como jornalero o en otras actividades (lavado, planchado, albañil y triciclero), lo utilizan para la compra de los alimentos que consumirán durante la semana (ver gráfico No. 5)

\section{El nivel de ingreso mensual en la familia.}

En la zona de estudio el $61 \%$ de la población encuestada tiene ingresos menores de C\$ 3000, el 28\% de C $\$ 3001$ a C $\$ 3800$, el $7 \%$ de $C \$ 3801$ a C $\$ 4500$, el 3\% de C\$ 4501 a C $\$ 5300,1 \%$ de C $\$ 5300$ a más. Esto significa que 35 familias encuestadas tienen ingresos menores de los 3000 córdobas al mes. Esto indica que los ingresos están por debajo del salario de $\mathrm{C} \$ 3014.41$ mínimo para los trabajadores del campo y no disponen de suficiente dinero para cubrir las necesidades del hogar, en el caso de las familias que tienen ingresos más altos es debido a que tiene un trabajo fijo (1 maestro) y 9 familias equivalente al $10 \%$ de las encuestadas reciben remesas de familiares que están fuera del país (ver gráfica No. 6).

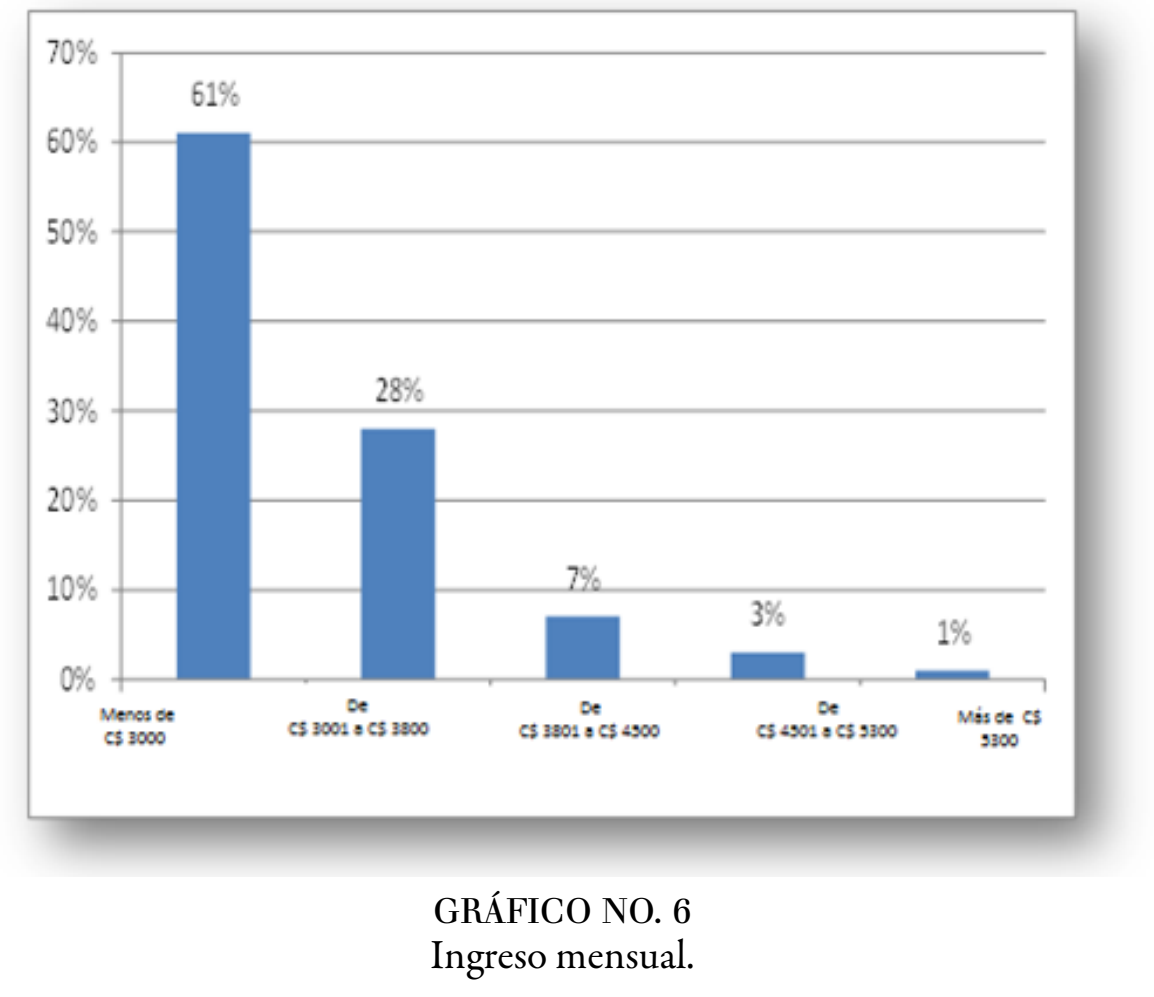

\section{Destino del ingreso mensual}

En los ingresos obtenidos mensualmente se encontró que el 38\% de los encuestados dedican para alimentación menos de 920 córdobas, el $47 \%$ dedican entre 921 a 1690 córdobas, el 12\% dedican entre 1691 a 2460 córdobas, el 1\% dedican entre 2461 a 3230 córdobas y un $2 \%$ dedican entre 3231 córdobas a más, la mayor parte de los ingresos lo destinan a la alimentación ya que con lo poco que obtienen únicamente pueden comprar los alimentos (ver gráfico No. 7). 


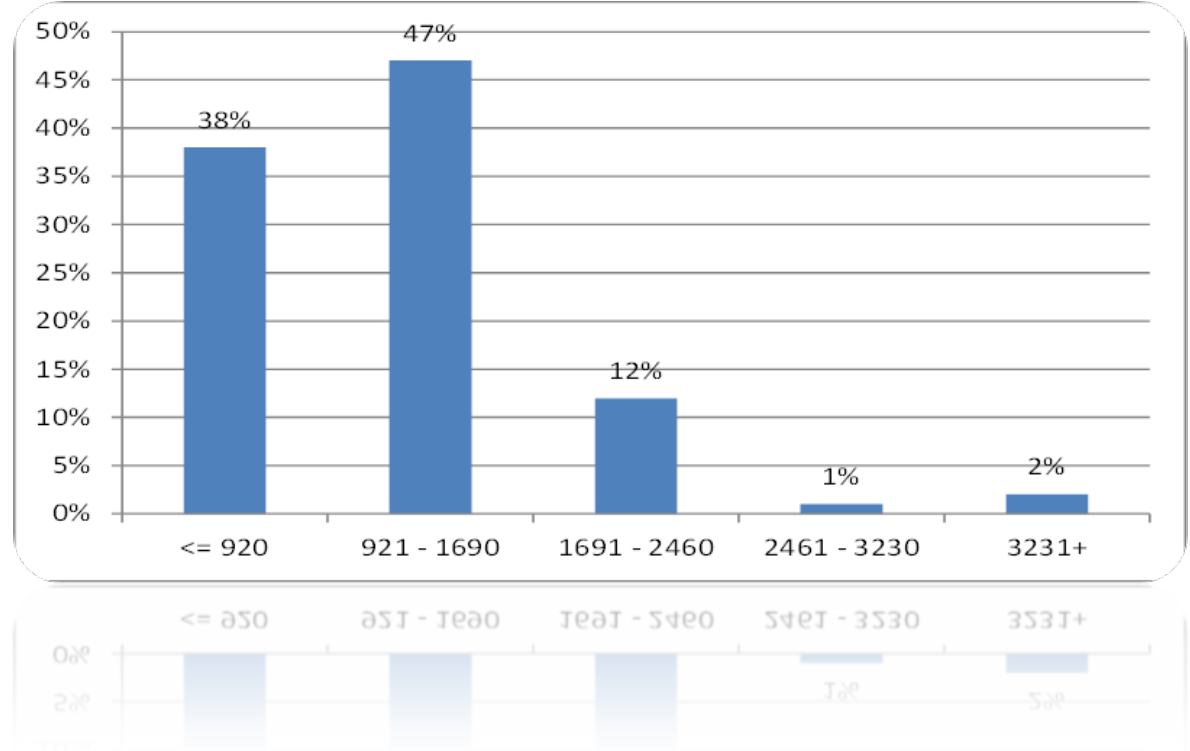

GRÁFICO NO. 7

Cantidad de Dinero que dedican a la alimentación

\section{Consumo}

Tipos de alimentos básicos en la alimentación de las familias.

Frecuencia porcentual de consumo de Cereales y granos básicos

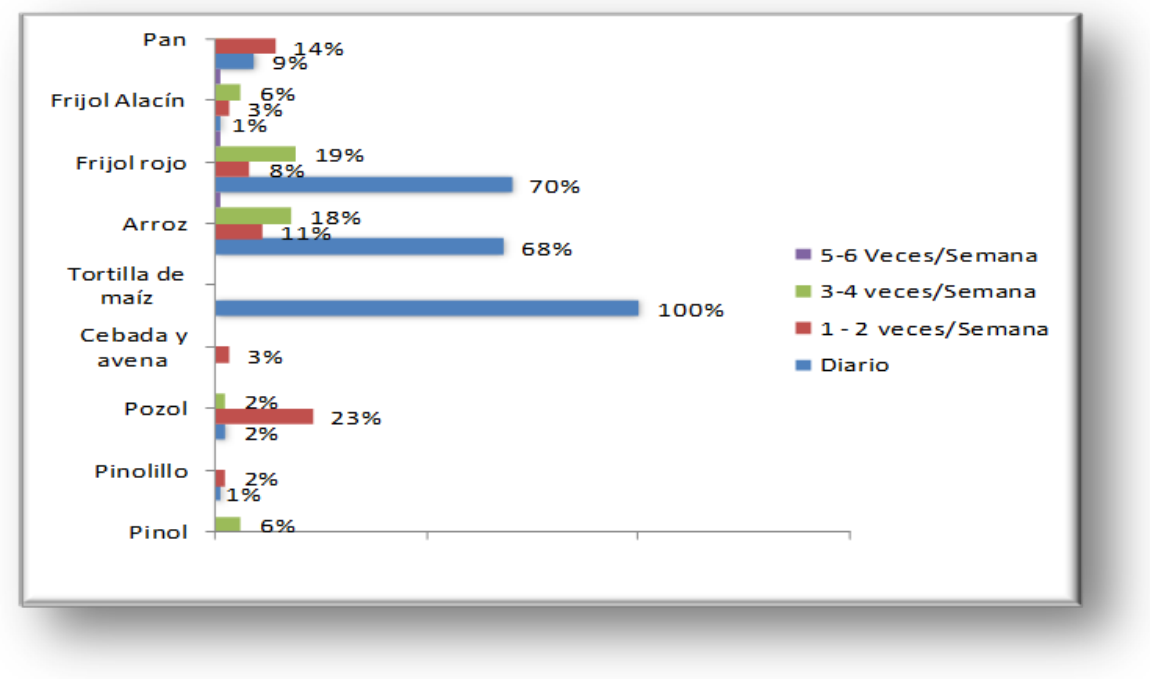

GRÁFICO NO. 8

Frecuencia de consumo de Cereales y granos básicos

En la frecuencia de consumo de cereales y grano básicos se encontró que consumen diariamente el 100\% de los encuestados tortilla de maíz, el 70\% frijol rojo, el 68\% arroz, el 9\% pan, el 2\% pozol, el 1\% frijol alacín y pinolillo. Con respecto a la frecuencia de consumo de una a dos veces por semana se encontró que el 23\% consumen pozol, el 14\% pan, el 11\% arroz, el $8 \%$ frijol rojo, el 3\% frijol alacín, el 3\% cebada y avena y el $2 \%$ pinolillo. En cuanto a la frecuencia de consumo de tres a cuatro veces por semana se encontró que el $19 \%$ consumen frijol rojo, el $18 \%$ arroz, el $6 \%$ frijol alacín, el $6 \%$ pinol y el $2 \%$ pozol y en lo referido a la 
frecuencia de consumo de cinco a seis veces por semana se encontró que el $1 \%$ consumen frijol alacín y pan. Esto probablemente se deba a que no todas las familias tienen la facilidad de poder accesar a estos productos y el patrón alimenticio está basado en el consumo de granos básicos consumiendo más tortilla de maíz, arroz y frijoles, además de los derivados del maíz que son los cereales en consumo de mínimas cantidades. (Ver gráfico No. 8).

\section{Comportamiento alimentario.}

Tiempos de comida.

En los tiempos de comida que realizan las familias encuestadas se encontró que el 59\% de los encuestados realizan los tres tiempos, el 22\% realizan dos tiempos desayuno y almuerzo, el 13\% realizan solo un tiempo el almuerzo debido a los pocos ingresos obtenidos y el 6\% realizan dos tiempos el almuerzo y cena. De estos los que consumen los 3 tiempos de comida son las familias que tienen mayor ingresos y tienen trabajos permanentes y algunas familias lo hacen de remesas familiares y los que solo hacen 2 tiempos de comida son las familias que solo consumen de lo poco que producen y de ayudas comunitarias y del gobierno y ONG. (Ver gráfico No. 9).

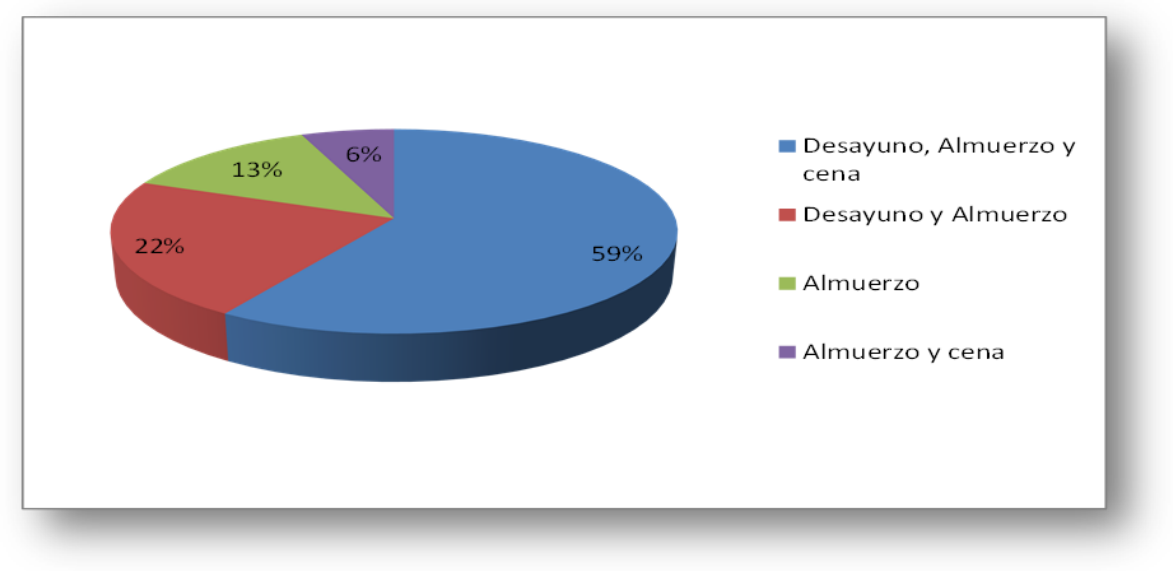

GRÁFICO NO. 9

Tiempos de comida realizado por las familias por día.

\section{Información Comercial de los alimentos}

Según los encuestados en la comunidad La Pavana se encontró que el 29\% compran los alimentos en pulpería, el $20 \%$ obtienen los alimentos en la pulpería y producción propia, el $17 \%$ en el mercado, el $14 \%$ en pulpería y puesto de ENABAS, el 9\% la obtienen de pulpería, puesto de ENABAS y producción propia, el 7\% obtienen en el mercado, pulpería y producción propia, el $2 \%$ en mercado y pulpería y el $1 \%$ obtiene los alimentos en el puesto de ENABAS (ver gráfico No. 10) 


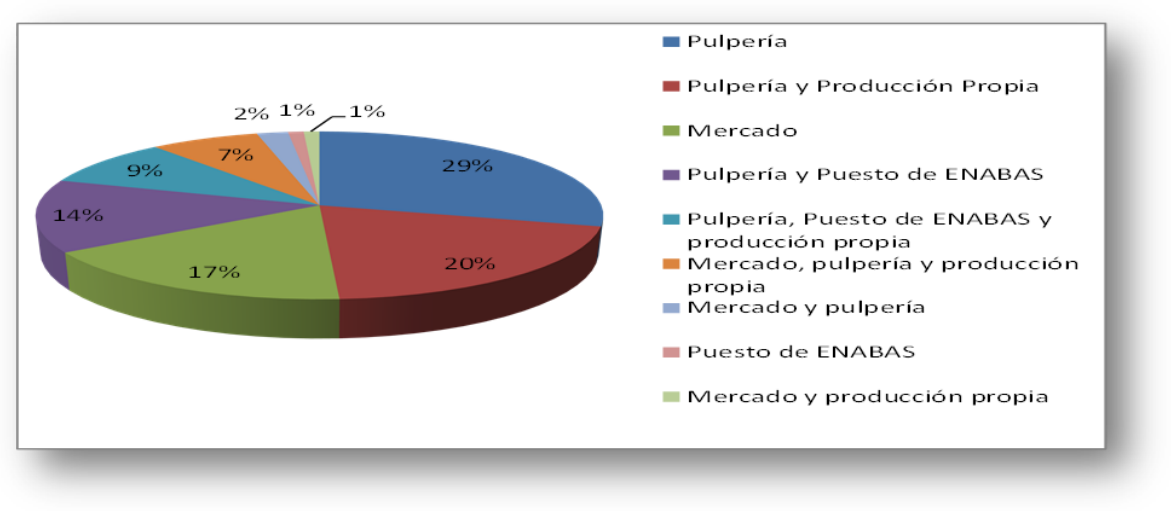

GRÁFICA NO. 10

Lugares donde compran los alimentos.

$$
\text { ENABAS }
$$

La mayor cantidad de las familias encuestadas compran los alimentos en las pulperías y no en las distribuidoras y mercados, debido a la larga distancia que existe de la comunidad a la cabecera municipal y es por eso que los obtienen a costos más altos.

\section{APROVECHAMIENTO BIOLÓGICO DE LOS ALIMENTOS}

\section{Servicios Básicos.}

Agua de consumo de los habitantes de la comunidad La Lavana

En el consumo de agua se encontró que el 100\% de los encuestados consumen agua de pozo ya que no cuentan con el servicio de agua potable en estos sectores cabe mencionar que en estos sectores, existen 53 pozos en total con una relación de 2 a 3 familias por pozos, algunas de las familias tienen que caminar distancias medias para la obtención del agua debido a que el caserío se encuentra disperso algunas tienen que cruzar la carretera para poder obtener el vital líquido lo cual pone en riesgo la seguridad de ellos. 


\section{Condiciones higiénicas ambientales}

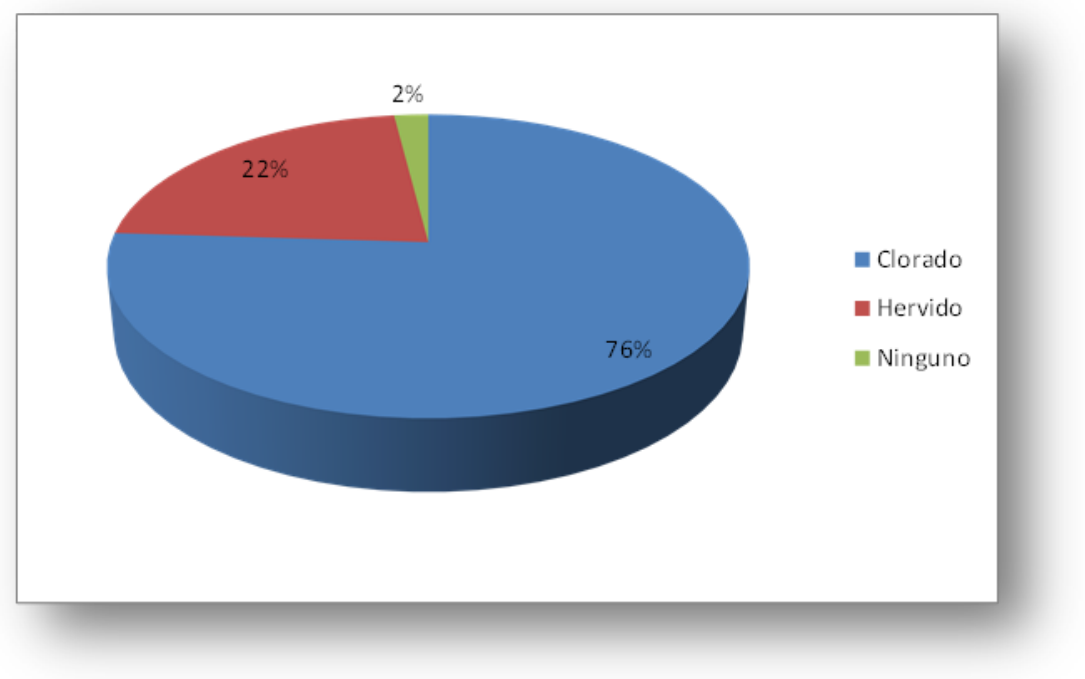

GRÁFICO NO 11

Tratamiento al agua de consumo en su hogar

En las condiciones higiénicas ambientales de las familias se encontró que el $76 \%$ de los encuestados cloran el agua de consumo, el 22\% hierven el agua y el 2\% no realizan ningún tratamiento (ver gráfico No. 11).

\section{Enfermedades más comunes presentadas en las familias.}

En las enfermedades más comunes se encontró que el 32\% de los encuestados padecen de gripe y tos debido a las condiciones ambientales predominantes en el medio, el 29\% fiebre, el 26\% diarrea a causa del consumo de alimentos no adecuados y el consumo de agua no tratada, el $4 \%$ neumonía asociadas también a factores condicionantes del ambiente en épocas de invierno y verano predominante mas en la época lluviosa, el 3\% padecen de presión arterial y problemas cardiaco, el $2 \%$ de parásitos mayor frecuencia de este problema en habitantes menores de edad ya que en ellos no predomina el habito de buena higiene y muchos de estos menores consumen alimentos de baja calidad e inocuidad, el $1 \%$ padecen de diabetes y el $1 \%$ no padece ninguna enfermedad, las enfermedades más comunes presentes en los habitantes de la comunidad se debe a factores climáticos, mal nutrición y de higiene personal (ver gráfico No. 12). 


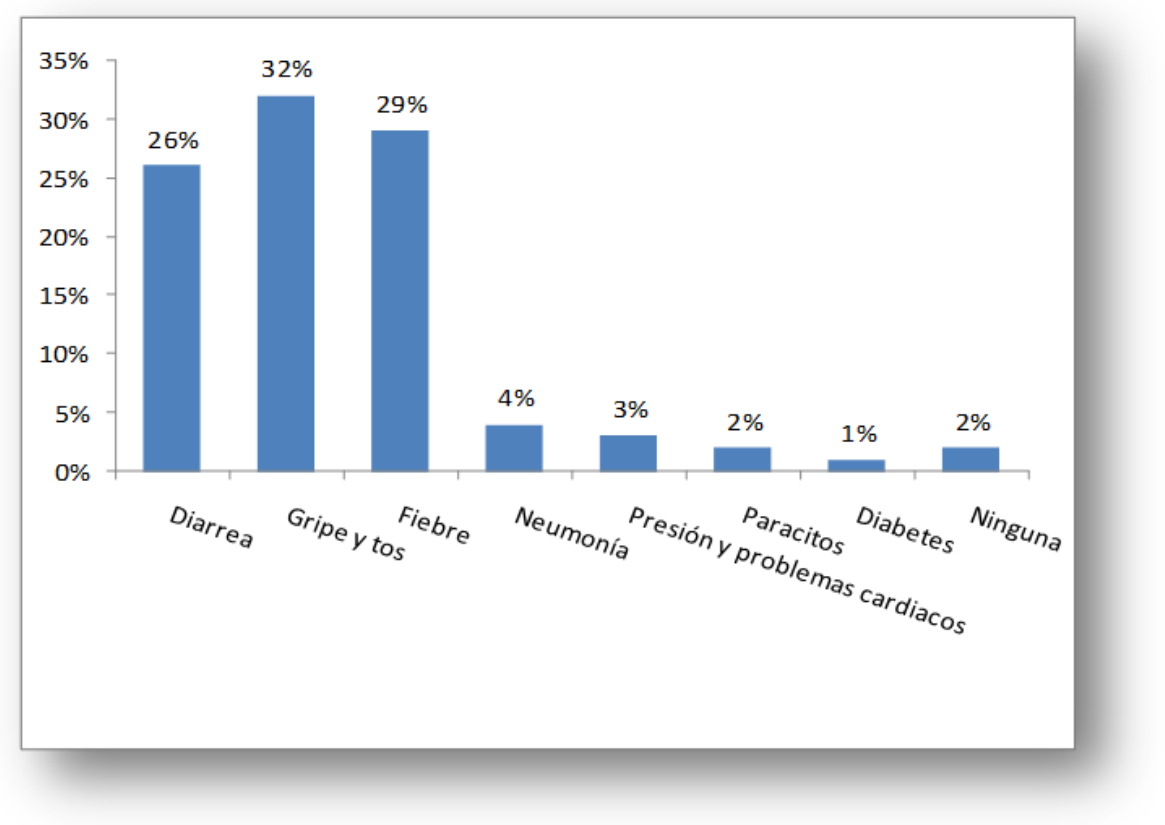

GRÁFICO NO. 12

Enfermedades más comunes.

\section{Lugares donde acuden en caso de enfermedades.}

Según los resultados de la encuesta realizada a los pobladores de la comunidad de la pavana sobre los centros a los cuales recurren cuando un miembro de la familia presenta una irregularidad en el estado de salud: Los lugares donde acuden las familias en caso de enfermedades que afecten o perjudiquen la salud en algún miembro del hogar según los encuestados se encontró que el $52 \%$ acuden al puesto de salud más cercano, el $21 \%$ acuden al puesto de salud La Pavana y hospital del municipio , el 16\% acuden al centro de salud, el 4\% acuden tanto al puesto de salud como a clínicas privadas, el 3\% hacen uso del hospital, el 2\% acuden a clínicas privadas y un $1 \%$ acuden al puesto de salud y centro de salud (ver gráfico No. 13). 


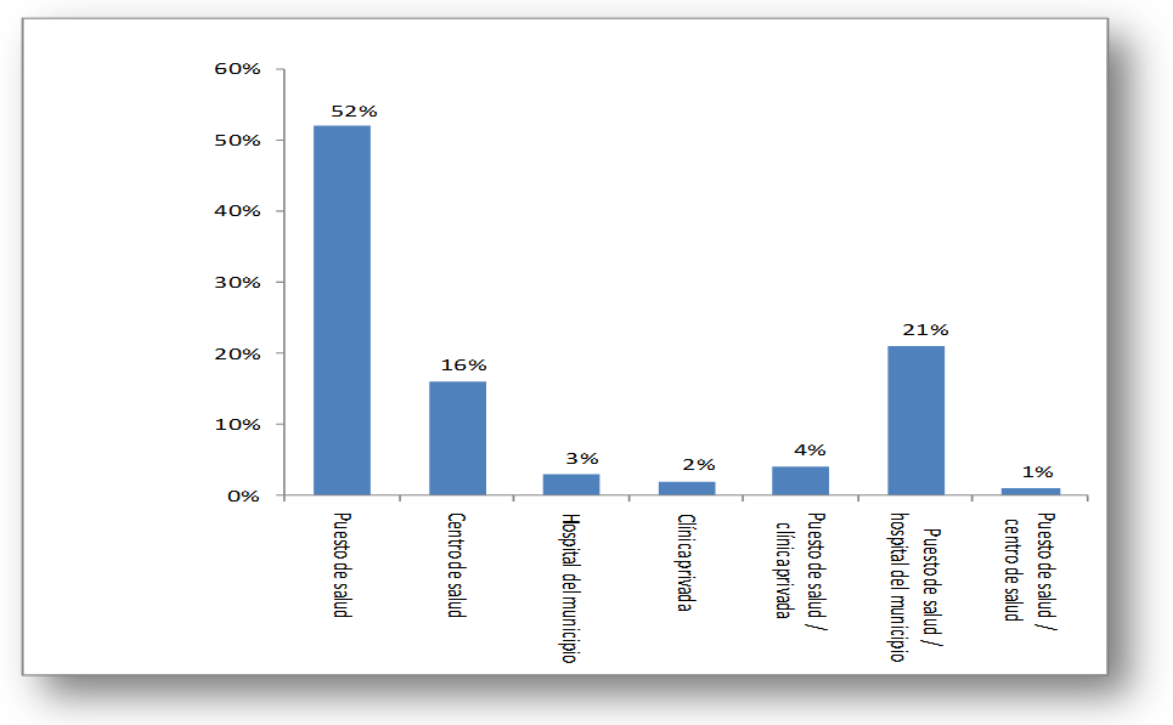

GRÁFICO NO. 13

Atención de la salud.

Según los resultados el 93\% de las familias encuestadas hacen uso de la atención en salud pública porque no tienen la capacidad económica para pagar un servicio privado ya que no poseen un trabajo estable y los que lo tienen el ingreso mensual anda por debajo del salario mínimo y solo un $7 \%$ usan tanto el servicio público como el privado estas familias son las que reciben remesas de familiares que se encuentran fuera del país y otros tienen un trabajo fijo y con un buen ingreso mensual.

\section{Estado nutricional}

Estado nutricional de los niños menores de 5 años (tabla No. 3).

TABLA NO.3

Estado nutricional de niños menores de 5 años en la comunidad La Pavana.

\begin{tabular}{lllll}
\hline Estado & \multicolumn{2}{l}{ Municipio de Somotillo } & \multicolumn{2}{c}{ Comunidad La Pavana } \\
\cline { 2 - 5 } Nutricional & cantidad & $\%$ & cantidad & $\%$ \\
\hline Sobrepeso & 47 & $0.4 \%$ & 15 & $1 \%$ \\
Normal & 10.291 & $94.1 \%$ & 1.337 & $86 \%$ \\
En riesgo & 468 & $4.3 \%$ & 151 & $10 \%$ \\
Desnutrido & 128 & $1.2 \%$ & 47 & $3 \%$ \\
TOTAL & 10.934 & $100 \%$ & 1.550 & $100 \%$ \\
\hline
\end{tabular}

MINSA y COMUSSAN de Somotillo.

Con referencia al estado nutricional de los niños menores de cinco años, según datos del Sistema local de atención en salud (SILAIS Chinandega) y la Comisión municipal de soberanía y seguridad alimentaria nutricional (COMUSSAN) se encontró que en el municipio de Somotillo el $0.4 \%$ de los niños presentan sobre peso, el $94.1 \%$ tienen peso normal, el $4.3 \%$ se encuentra en riesgo y el $1.2 \%$ de ellos están desnutridos. En el caso específico de la comunidad La Pavana se encontró que el 1\% de los niños tienen sobre peso, el 86\% se encuentran en peso normal, el $10 \%$ se encuentran en riesgo nutricional y un $3 \%$ de estos están desnutridos, 
lo que indica que las tazas de desnutrición es mayor en la comunidad La pavana que a nivel de municipio, de igual manera ocurre con los niños que están en riesgo y en sobre peso, otro aspecto importante es que del 100\% de los niños desnutridos existentes en el municipio el 37\% están en la comunidad La Pavana, del 100\% de los niños que están en riesgo el 32\% viven en la comunidad los balcones, y del 100\% de los niños que están en sobre peso en el municipio el 32\% se encuentran en la comunidad la pavana. A manera de resumen del $100 \%$ de los niños que tienen problemas de desnutrición (Sobre peso, en riesgo y desnutridos) en el municipio equivalente a 643 niños, el 33\% (213) de ellos viven en la comunidad la pavana y sus sectores aledaños.

\section{Conclusiones}

Con la recopilación de información a través de las encuestas realizadas a las familias de la comunidad la pavana y sus sectores aledaños se identificó que existe inseguridad alimentaria ya que el $79 \%$ de las familias no cuentan con tierra propia, además los productores no poseen tecnologías apropiadas tales como semillas mejoradas y obras de conservación de suelo en sus pequeñas parcelas, de manera que puedan poseer suelos productivos y garantizar una buena cosecha sin el consumo excesivo de insumos para la producción de sus cultivos lo cual los hace vulnerables en la disponibilidad de los alimentos.

En la comunidad no existe extensión de tecnologías que promuevan las capacidades productivas por esta razón las familias no consumen una diversidad de alimentos ya que los productores han adoptado el monocultivo en sus pequeñas parcelas, limitándose a diversificarla y a darle un mejor provecho al espacio. como consecuencia de este evento la tenencia de la tierra es el factor principal, seguido de la cultura, conocimiento y poco interés de los productores que poseen tierra propia, esto se ve reflejado en un $93 \%$ de los productores que no establecen ningún tipo de hortalizas para mejorar la dieta alimenticia de sus familias.

Las condiciones climáticas en la zona son variables y ha venido disminuyendo la cantidad de precipitaciones anuales la cual ha afectado negativamente los rendimientos productivos y la diversidad de alimentos.

A pesar de que existen políticas institucionales dirigidas al sector agropecuario tal como es el programa CRISSOL (cristiano, Socialista y Solidario), los productores no pueden optar a este programa por que no cumplen con ciertos criterios tal como es la tenencia de la tierra ya que el $79 \%$ no cuenta con tierra propia y los que poseen tierra no tienen un nivel de organización que les permita accesar a estos programas de gobierno.

En la comunidad no existen fuentes de empleo por lo tanto las familias se ven obligadas a realizar trabajos en la cabecera municipal y los ingresos obtenidos diariamente por las familias son aproximado al requerimiento económico mínimo de subsistencia de \$2/día establecido para familias que viven en línea de pobreza, las familias están obteniendo ingresos promedios de 2.60 dólares por día, lo que demuestra que están por debajo del salario mínimo que es de $C \$ 3014.42$ para trabajadores del campo, razón por la cual tienen menos acceso y menor capacidad para la compra de sus alimentos.

El nivel educativo en la comunidad es muy bajo, ya que la situación económica no les permite culminar una carrera profesional o técnica, esto ha limitado la profesionalización de jóvenes los cuales al terminar su secundaria se insertan al campo laboral informal, para garantizar alimentos a la familia.

Los rendimientos promedios de los cultivos maíz, sorgo y ajonjolí establecidos en la comunidad andan por debajo del promedio nacional de la zona seca de Nicaragua, estos rendimientos productivos no satisfacen la demanda de las familias, lo que se ve reflejado en los tiempos de comida ya que solo el $59 \%$ de las familias realizan tres tiempos de comida.

El patrón alimentario de las familias de la comunidad la pavana está compuesto por 9 alimentos de 21 alimentos que conforman el patrón alimenticio en el departamento de Chinandega, la dieta está centrada en el consumo de carbohidratos debido a esto existe un 3\% de desnutrición de los niños en la comunidad según datos del MINSA. 
Las familias de la comunidad la Pavana tienen un déficit en los servicios básicos ya que solo el $92 \%$ poseen letrinas para las necesidades fisiológicas, no cuentan con servicio de agua potable, además no existe un buen manejo de los desechos sólidos y líquidos lo que provoca contaminación del medio ambiente y la propagación de enfermedades diarreicas con un 26\% y virales con el $61 \%$ de acuerdo a datos del MINSA.

Las familias encuestadas no gozan de un techo digno ya que el $34 \%$ no poseen una vivienda propia, debido a que viven en casas prestadas y el $66 \%$ cuentan con viviendas propias y estas se encuentran en terrenos de los padres que les prestan el solar para la construcción de las mismas.

Los pilares que inciden en la inseguridad alimentaria en la comunidad la pavana son la disponibilidad y acceso de los alimentos, ya que se encuentran bien marcados tres factores de cada uno de ellos.

\section{Recomendaciones}

Los desafíos para el mejoramiento de la Seguridad Alimentaria y Nutricional son múltiples y deben ser apropiados por todos, se requiere de esfuerzos sostenibles que sean coherentes con políticas y estrategias a mediano y largo plazo del consenso entre el sector municipal, sin olvidar la responsabilidad del Estado tanto a nivel central como local.

1- Promover proyectos de planes de negocios tales como: pulperías, y crianza de aves de patio que generen fuentes de empleo para que las familias puedan mejorar sus ingresos.

2- Impulsar la producción de alimentos y consumo mediante la implementación huertos de patio. para la producción de hortalizas.

3- Promover por medio del MINED el establecimiento de huertos escolares que ayuden al mejoramiento de la merienda escolar de los niños, así como también el involucramiento de ellos a la producción de huertos.

4- Coordinar con instituciones como el INTA, FH, alcaldía, ADESS, NITLAPLAN, CIPRES, MEFCCAC y el MAG que están presentes en el municipio para que estas trabajen en pro de alternativas que puedan mejorar los rendimientos productivos de las familias que tienen el acceso a cultivar en la comunidad.

5- Promover a través del MINSA, Alcaldía, INTA, COMUSSAN y FH y universidades locales capacitaciones y actividades comunitarias para el rescate de la cultura alimentaria autóctona de la comunidad, así como también campañas ambientales de sensibilización y proyectos de agua y saneamiento de la comunidad.

6- Que los programas de gobierno presentes en el municipio lleguen a la comunidad y puedan beneficiar a las personas más vulnerables de los sectores aislados.

7- Que se establezcan relaciones entre las instituciones existentes en el municipio para llevar a cabo proyectos de extensión rural para el desarrollo comunitario de tecnologías apropiadas y accesible para los pequeños productores de la comunidad.

\section{Literatura Citada}

Amuninic. (2012). Programa de fortalecimiento a la Gobernabilidad Local para el Objetivo de Desarrollo del Milenio. Guia dirigida a tecnicos municipales para la integracion del enfoque de seguridad alimentaria y nutricional en la gestion del desarrollo local, 90 .

Bonilla Madrigal, J. (2001). Politica de Seguridad Alimentaria y Nutricional. Managua: ENCOR.

Brunetti, A., \& Masci, E. (2009). Manual para la evaluacion de Seguridad alimentari en emergencia. Italia: Programa Mundial de Alimento.

Delgadilllo Paguaga, N. (2004). Analisis de pobreza y la Seguridad Alimentaria Nutricional en Nicaragua. Managua: Programa MECOVI.

FAO. (206). Informe de Politicas de Seguridad Alimentaria . 
Silvio Javier Sáenz Rojas, et al. Evaluación del impacto de los Pilares de la Seguridad y Soberaní...

Hernández Sampieri, R., Fernández Collado, C., \& Baptista Lucio, P. (2006). Metodología de la Investigación. Iztapalapa: McGraw Hill.

MAGFOR. (2009). Direccion General de Politica Agropecuaria y Forestal. Politica de Seguridad Y Soberania Alimentaria y Nutricional desde el sector Publico y Rural , 48.

Ramos, L. A., Giraldo, M., Castello, L. M., Gonzalez, H., \& Celiz, M. L. (2008). Relatos Historicos de la Seguridad Alimentaria y Reconocimiento de las condiciones en nuestro entorno. La Seguridad Alimentaria y Nutricional en las Escuelas de Campo de Agricultores, 40.

FAO. (2009). Marco nacional de prioridade para la asistencia tecnica de la FAO. Managua.

Paola, H. (2008). Nutricion y seguridad alimentaria en la comunidad Quillotoru, Canton, Guamote Provincia de Chimborazo. Chimborazo.

PMA. (2009). Manual para la evaluacion de la seguridad alimentaria en emergencia . Roma, Italia. 
Revista Iberoamericana de Bioeconomía y Cambio Climático, 2018, 4(8), Agosto-Diciembre, ISSN: 2410...

\section{Anexos}

TABLA 4

Operacionalización de las variables, disponibilidad, consumo, acceso y aprovechamiento biológico.

\begin{tabular}{|c|c|c|c|}
\hline Variable & Sub variable & Indicador & Escala \\
\hline \multirow[t]{4}{*}{ Disponibilidad } & $\begin{array}{l}\varnothing \text { Disponibilidad de } \\
\text { los alimentos }\end{array}$ & $\begin{array}{l}\varnothing \text { Tenencia de la tierra } \\
\varnothing \text { Tipos de actividad } \\
\text { económica que realiza en } \\
\text { el ciclo agrícola. } \\
\varnothing \text { Épocas de siembra. } \\
\varnothing \text { Producción agrícola } \\
\varnothing \text { Cantidad de tierra } \\
\text { destinada a la agricultura } \\
\varnothing \text { Tipos de rubros que se } \\
\text { establecen en la } \\
\text { comunidad La Pavana. } \\
\varnothing \text { Destino de la. } \\
\text { producción. } \\
\text { Consumo/Venta } \\
\varnothing \text { Producción forestal. } \\
\varnothing \text { Disponibilidad de agua } \\
\text { para uso agrícola } \\
\varnothing \text { Existencia de ganado } \\
\text { mayor. } \varnothing \text { Existencia de } \\
\text { ganado menor. }\end{array}$ & Ordinal \\
\hline & $\begin{array}{l}\varnothing \text { Producción } \\
\text { Interna de } \\
\text { alimentos. }\end{array}$ & $\begin{array}{l}\varnothing \text { Rendimiento por } \\
\text { manzana de granos } \\
\text { básicos (Maiz, frijol, } \\
\text { sorgo etc) } \varnothing \text { Rendimiento } \\
\text { por área de hortalizas } \\
\text { (pipián, Ayote, Tomate), } \\
\varnothing \text { Destinos de productos } \\
\text { pecuarios leche, cuajada, } \\
\text { huevos, ganado bovino y } \\
\text { aves. }\end{array}$ & Ordinal \\
\hline & $\varnothing$ Vías de acceso & $\begin{array}{l}\varnothing \text { Tipo de transporte que } \\
\text { Sutiliza. }\end{array}$ & Ordinal \\
\hline & $\begin{array}{l}\varnothing \text { Atención a la } \\
\text { seguridad } \\
\text { alimentaria. }\end{array}$ & $\begin{array}{l}\text { D Cantidad de familia } \\
\text { que reciben ayuda } \\
\text { alimentaria }\end{array}$ & Ordinal \\
\hline \multirow[t]{2}{*}{ Acceso } & $\begin{array}{l}\varnothing \text { Técnicas de } \\
\text { obtención de los } \\
\text { alimentos }\end{array}$ & $\begin{array}{l}\varnothing \text { Frecuencia de } \\
\text { realización de compra de } \\
\text { alimentos. } \varnothing \text { Nivel de } \\
\text { ingreso mensual de la. } \\
\text { familia. } \varnothing \text { Situación } \\
\text { laboral de los miembros } \\
\text { de las familias en estudio. } \\
\varnothing \text { Destino del ingreso } \\
\text { mensual en la familia. }\end{array}$ & Ordinal \\
\hline & $\varnothing$ Almacenamiento & $\begin{array}{l}\varnothing \text { Técnicas de } \\
\text { almacenamiento de los } \\
\text { alimentos }\end{array}$ & Ordinal \\
\hline \multirow[t]{4}{*}{ Consumo } & $\begin{array}{l}\varnothing \text { Tipos de } \\
\text { alimentos básicos en } \\
\text { la alimentación de } \\
\text { las familias. }\end{array}$ & $\begin{array}{l}\varnothing \text { Frecuencia porcentual } \\
\text { de consumo de cereales y } \\
\text { granos básicos. } \\
\varnothing \text { Frecuencia porcentual } \\
\text { de consumo de lácteos } \\
\varnothing \text { Frecuencia porcentual } \\
\text { de consumo de carnes y } \\
\text { sustitutos } \varnothing \text { Frecuencia } \\
\text { porcentual de consumo } \\
\text { de Misceláneos } \\
\varnothing \text { Frecuencia porcentual } \\
\text { de consumo de frutas y } \\
\text { verduras. }\end{array}$ & Ordinal \\
\hline & $\begin{array}{l}\varnothing \text { Suficiencia del } \\
\text { consumo alimentos. }\end{array}$ & $\begin{array}{l}\varnothing \text { Patrón Alimenticio. } \\
\varnothing \text { Comportamiento } \\
\text { alimentario. } \varnothing \text { Educación } \\
\text { alimentaria y nutricional. } \\
\varnothing \text { Nivel educativo. }\end{array}$ & Ordinal \\
\hline & $\begin{array}{l}\varnothing \text { Información } \\
\text { Comercial de los } \\
\text { alimentos }\end{array}$ & $\begin{array}{l}\varnothing \text { Información de lugares } \\
\text { donde obtener alimentos. } \\
\varnothing \text { Medios por los cuales } \\
\text { llega la publicidad. }\end{array}$ & Ordinal \\
\hline & $\begin{array}{l}\varnothing \text { Composición del } \\
\text { hogar y la familia. }\end{array}$ & $\begin{array}{l}\varnothing \text { Rangos de edad y sexo } \\
\text { de la familia. }\end{array}$ & Ordinal \\
\hline \multirow[t]{2}{*}{$\begin{array}{l}\text { Aprovechamiento } \\
\text { biológico de los } \\
\text { Alimentos }\end{array}$} & $\begin{array}{l}\varnothing \text { Servicios } \\
\text { Básicos. }\end{array}$ & $\begin{array}{l}\varnothing \text { Tenencia de vivienda. } \\
\varnothing \text { Infraestructura de los } \\
\text { hogares. } \varnothing \text { Agua de } \\
\text { consumo. } \varnothing \text { Condiciones } \\
\text { higiénicas ambientales: } \\
\text { Manejo del agua, } \\
\text { Necesidades fisiológicas, } \\
\text { Tratamiento de la basura. } \\
\varnothing \text { Tipo de iluminación de } \\
\text { la vivienda } \\
\varnothing \text { Enfermedades más } \\
\text { comunes presentadas en } \\
\text { las familias. } \varnothing \text { Lugares } \\
\text { donde acuden los } \\
\text { miembros de la familia en } \\
\text { casa de enfermedades. }\end{array}$ & $\begin{array}{l}\text { Ordinal - } \\
\text { nominal }\end{array}$ \\
\hline & $\begin{array}{l}\varnothing \text { Estado } \\
\text { Nutricional. }\end{array}$ & $\begin{array}{l}\varnothing \text { Estado nutricional de } \\
\text { los niños menores de } 5 \\
\text { años. }\end{array}$ & Ordinal \\
\hline
\end{tabular}


Silvio Javier Sáenz Rojas, et al. Evaluación del impacto de los pilares de la SEguridad y Soberaní... 